\title{
Repeated Moral Hazard with Effort Persistence
}

\author{
Arantxa Jarque $^{1}$ \\ Departamento de Fundamentos del Analisis Economico \\ Universidad de Alicante \\ Ctra. San Vicente del Raspeig, s/n, Alicante 03080, Spain. \\ Email: jarque@merlin.fae.ua.es. \\ January 28, 2005
}

${ }^{1}$ I would like to thank Marina Azzimonti, Hal Cole, Juan Carlos Hatchondo, Leonardo Martínez, Iv́an Werning and especially Hugo Hopenhayn for very useful suggestions and discussions. I gratefully acknowledge financial support from the Banco de España and the hospitality of the Universidad Torcuato di Tella, were this research was started. 


\begin{abstract}
We study a problem of repeated moral hazard where the effect of effort is persistent over time: each period's outcome distribution is a function of a distributed lag of past efforts. We study a particular specification of persistence: the distribution of the outcomes depends on the sum of discounted efforts. We show that when the utility of the agent is linear in effort, a simple rearrangement of terms in his lifetime utility translates this problem into a standard repeated moral hazard. As a consequence, the optimal consumption profile is observationally equivalent to that of a related problem without persistence. This suggests that the presence of persistence does not necessarily change the properties of optimal contracts.

Journal of Economic Literature Classification Numbers: D30, D31, D80, D82.

Key Words: mechanism design; repeated agency.
\end{abstract}




\section{Introduction}

In many real life situations, the actions that agents take at a point in time have long lasting consequences, affecting the productivity of their future actions. Such persistence must be taken into account in a repeated agency relationship when designing the optimal contract. A health insurance provider knows that unhealthy habits throughout the years predetermine its clients

health to be very weak, making later efforts to preserve it rather ineffective. In any kind of job in which there is learning by doing there is an obvious interest in making the agent work hard in the initial periods. The common feature of these environments is that current effort has an effect on the distribution of current and future outcomes. This persistence is a key difference between this model and the standard repeated moral hazard problem.

Without persistence, outcome realizations are independent of previous actions, making incentives to deviate in a given period depend only on the continuation contract. Although the contract depends on past history, all the relevant information can be summarized in one variable, the continuation utility promised to the agent in each period. This feature makes it possible to write the repeated moral hazard in a recursive way (see [10]), simplifying the analysis and computation of the optimal contract. There is an extensive literature that studies the characteristics of efficient allocations of consumption and work effort in an economy with moral hazard (see [2],[8] and [6]). Introducing persistence of effort is not a straightforward generalization since the methods used for the standard case do not easily extend to this setup. So far there is no clear understanding of how the characteristics of the optimal consumption 
path change with the presence of persistence.

Fernandes and Phelan [3] provide the first recursive treatment of agency problems with effort persistence. In their paper, the current effort of the agent affects output in the same period and in the following one. Their setup is characterized by three parameters: the number of periods that the effect of effort lasts for, the number of possible effort levels and the number of possible outcome realizations. All three parameters are set to two and this makes their formulation and their computational approach feasible. The curse of dimensionality applies whenever any of the three parameters is increased. Moreover, no results are given in their paper on how the properties of the optimal contract differ from the case without persistence.

Mukoyama and Sahin [5] show in a two period contract with two possible levels of effort that if persistence is high it may be optimal for the principal to perfectly insure the agent in the first period. Our results imply that their result will not hold in general when allowing for a continuum of effort choices.

In this paper we show that whenever the utility of the agent is linear in effort and each period's outcome distribution is a function of a distributed lag of past efforts, persistence is not a major complication. With a simple rearrangement of the terms in the expression for the lifetime utility of the agent, we show that the optimal sequence of consumption and effort recommendations can be easily recovered from the solution of a related standard repeated moral hazard. The sequence of contingent consumptions is a solution for both the auxiliary and the original problem, making them observationally equivalent. This means that, under 
our assumptions, the optimal sequences of consumption in problems with persistence have the same properties as the ones in problems without persistence, and the results found so far in the literature apply also to the more realistic setup studied here. Persistence, however, induces the principal to front load most of the effort requirements in the first period, asking the agent to simply provide little increments in the following periods to compensate for the diminishing effect of the initial effort.

\section{Model}

Consider a problem of repeated moral hazard where the effort carried out by the agent each period affects current and future output distributions. Assume the agent has additively separable utility that is linear in effort:

$$
U\left(c_{t}, e_{t}\right)=u\left(c_{t}\right)-e_{t}
$$

where $c_{t}$ is consumption, with $c_{t} \in \mathbb{R}_{+} \forall t$, and $e_{t}$ denotes effort, with $e_{t} \in \mathbb{R} \forall t$. Assume $u$ is strictly concave.

Let $Y_{t}=Y=\left\{y_{1}, y_{2}, \ldots, y_{n}\right\}$ be the set of possible outcomes each period. Let $Y^{t}=\Pi_{\tau=0}^{t} Y_{\tau}$, with typical element $y^{t}=\left(y_{0}, y_{1}, \ldots, y_{t}\right)$, denote the set of histories of outcome realizations up to time $t$. The history of outcomes is assumed to be common knowledge. To capture the persistence of effort, the distribution of output is a function of all past efforts, $e^{t}=e_{1}, e_{2}, \ldots, e_{t}$, and is denoted by $\pi\left(y_{i} \mid e^{t}\right)$. We assume $\pi\left(y_{i} \mid e^{t}\right)>0$ for all $y_{i} \in Y$ and all $e^{t}$. 
A contract is a tuple

$$
\mathbf{C} \equiv\left(\left\{e_{t}\left(y^{t-1}\right)\right\}_{t=1}^{\infty},\left\{c_{t}\left(y^{t}\right)\right\}_{t=0}^{\infty}\right)
$$

At period zero, the principal chooses the level of effort to be implemented at each point in time and for each possible history of outcomes. Consumption is given as a contingent transfer to the agent in order to provide the necessary incentives.

The timing is as follows: at time zero, the agent accepts or rejects the contract offered by the principal. If he accepts, at the beginning of any period $t$ he chooses the amount of effort he wants to exert. Output $y_{t}$ is realized according to the distribution determined by the effort choices up to time $t$, and the corresponding amount of consumption $c_{t}\left(y^{t}\right)$ is given to the agent.

Let $\Pi\left(y^{t} \mid e^{t}\left(y^{t-1}\right)\right)=\prod_{\tau=1}^{t} \pi\left(y_{i} \mid e^{\tau}\left(y^{\tau-1}\right)\right)$ denote the probability of history $y^{t}$ for a given sequence of functions $\left\{e_{\tau}\left(y^{\tau-1}\right)\right\}_{\tau=1}^{t}$. Let $E U(\mathbf{C})$ denote the expected utility of the agent from a given contract $\mathbf{C}$, which is calculated in the following way:

$$
E U(\mathbf{C})=\sum_{t=1}^{\infty} \sum_{y^{t}} \beta^{t-1}\left\{u\left(c_{t}\left(y^{t}\right)\right)-e_{t}\left(y^{t-1}\right)\right\} \Pi\left(y^{t} \mid e^{t}\left(y^{t-1}\right)\right)
$$

To complete the description of the problem, we now turn to the principal's preferences and objective function. Assume the principal is risk neutral and designs the contract $\mathbf{C}$ in order to maximize the expected discounted cash flow given by the difference between the output and the promised contingent payments:

$$
\max _{\left\{e_{t}\left(y^{t-1}\right)\right\}_{t=1}^{\infty},\left\{c_{t}\left(y^{t}\right)\right\}_{t=0}^{\infty}} \sum_{t=1}^{\infty} \sum_{y^{t}} \beta^{t-1}\left\{y_{t}-c_{t}\left(y^{t}\right)\right\} \Pi\left(y^{t} \mid e^{t}\left(y^{t-1}\right)\right) .
$$

As usual, the principal faces participation and incentive constraints. The Participation Con- 
straint (PC) makes sure the initial expected utility of the agent in the contract is at least as large as his outside utility $\underline{U}$ :

$$
E U(\mathbf{C}) \geq \underline{U}
$$

The Incentive Constraint (IC) makes sure that the agent does not have a profitable deviation, i.e. the sequence of effort levels recommended by the principal must be a solution to the maximization problem faced by the agent for the contract $\mathbf{C}$; namely

$$
\left\{e_{t}\left(y^{t-1}\right)\right\}_{t=1}^{\infty} \in \arg \max _{\left\{\widehat{e}_{t}\left(y^{t-1}\right)\right\}_{t=1}^{\infty}} E U(\mathbf{C})
$$

The set of possible deviations of the agent is extremely large, given by sequences of functions mapping histories of outcomes into levels of effort. Unlike in a standard repeated moral hazard problem, there is no recursive representation making the IC a per-period constraint on the level of effort. Persistence means that, for a given continuation contract, incentives for deviation at a certain period may depend on the actual sequence of efforts chosen by the agent up to that point. In consequence, we need to check for the possibility of joint deviations involving effort choices in more than one period; this complicates the computation of the optimal contract.

However, as we now show, the problem with persistence can be translated into a standard repeated moral hazard, where the usual recursive tools can be used to derive the optimal contract. ${ }^{1}$ In order to do this, we assume the distribution of outcomes depends on the depreciated

\footnotetext{
${ }^{1}$ Even without the assumption of linear disutility of effort there exists a recursive formulation of the problem. However, the characterization of the problem becomes complicated due to the fact that the value function of the principal depends on three state variables. Carrying extra state variables makes the preferences of the agent
} 
sum of past efforts. Let $\delta \in(0,1)$ be the rate at which the effect of effort on the distribution of outcomes decreases. For a sequence $e^{t}=\left(e_{\tau}\right)_{\tau=1}^{t}$, let $s_{t}$ denote the depreciated sum of past efforts, i.e.:

$$
s_{t}=\sum_{\tau=1}^{t}(1-\delta)^{t-\tau} e_{\tau} .
$$

The probability distribution over the outcomes is given by $\pi\left(y_{t} \mid s_{t}\right)$. We use $s^{t}$ to denote the history up to time $t$. The results in this paper are valid whenever the dependence of $s_{t}$ on the history of efforts is linear, so that the effect of $e_{t}$ in any future period can be accounted for in $t$ independently of the values of future variables. In particular, the value of the depreciation factor $\delta$ could be changing over time and the result would still hold.

With this assumption the problem of the principal can be restated in terms of this sum of discounted efforts, which is the variable that effectively determines the distribution of the outcomes:

$$
\sum_{t=1}^{\infty} \sum_{y^{t}} \beta^{t-1}\left\{y_{t}-c_{t}\left(y^{t}\right)\right\} \Pi\left(y^{t} \mid s^{t}\left(y^{t-1}\right)\right) .
$$

Since $s_{t}$ is a linear combination of past efforts we can also write the expected utility of the agent in terms of this variable. Given that $e_{t}=s_{t}-(1-\delta) s_{t-1}$, we now rewrite the expected over continuation contracts known on the equilibrium path. This is done in the same spirit as in the recent papers analyzing moral hazard problems with unobserved savings (see Á. Ábrahám and N. Pavoni: "Efficient Allocations with Moral Hazard and Hidden Borrowing and Lending." Mimeo, University College London (2003) and I. Werning: "Moral Hazard with Unobserved Endowments: A Recursive Approach." Mimeo, University of Chicago (2001)). 
utility as: ${ }^{2}$

$$
\sum_{t=1}^{\infty} \sum_{y^{t}} \beta^{t-1}\left\{u\left(c_{t}\left(y^{t}\right)\right)-s_{t}\left(y^{t-1}\right)+(1-\delta) s_{t-1}\left(y^{t-2}\right)\right\} \Pi\left(y^{t} \mid s^{t}\left(y^{t-1}\right)\right) \quad\left(E U(\mathbf{C})^{\prime}\right)
$$

Given the linearity of the disutility function, a rearrangement of terms in the expression for the expected utility in the $\mathrm{PC}$ and the IC comes then very naturally, simplifying the problem enormously: the terms in which an $s$ with the same time index enters the expression above can be collected into one:

$$
\sum_{t=1}^{\infty} \sum_{y^{t}} \beta^{t-1}\left\{u\left(c_{t}\left(y^{t}\right)\right)-(1-\beta(1-\delta)) s_{t}\left(y^{t-1}\right)\right\} \Pi\left(y^{t} \mid s^{t}\left(y^{t-1}\right)\right) \quad\left(E U(\mathbf{C})^{\prime \prime}\right)
$$

Now the problem of the principal is:

$$
\begin{gathered}
\max _{\left\{s_{t}\left(y^{t-1}\right)\right\}_{t=1}^{\infty},\left\{c_{t}\left(y^{t}\right)\right\}_{t=0}^{\infty}} \sum_{t=1}^{\infty} \sum_{y^{t-1}} \beta^{t-1}\left\{y_{t}-c_{t}\left(y^{t}\right)\right\} \Pi\left(y^{t} \mid s^{t}\left(y^{t-1}\right)\right), \\
\text { s.t. } \\
\underline{U}=\sum_{t=1}^{\infty} \sum_{y^{t}} \beta^{t-1}\left\{u\left(c_{t}\left(y^{t}\right)\right)-(1-\beta(1-\delta)) s_{t}\left(y^{t-1}\right)\right\} \Pi\left(y^{t} \mid s^{t}\left(y^{t-1}\right)\right) \\
\left\{s_{t}(\cdot)\right\} \in \arg \max _{\left\{s_{t}(\cdot)\right\}} \sum_{t=1}^{\infty} \sum_{y^{t}} \beta^{t-1}\left\{u\left(c_{t}\left(y^{t}\right)\right)-(1-\beta(1-\delta)) s_{t}\left(y^{t-1}\right)\right\} \Pi\left(y^{t} \mid s^{t}\left(y^{t-1}\right)\right)
\end{gathered}
$$

By simple inspection of the above problem it becomes apparent that the solution for the optimal sequence of $s_{t}$ is also a solution for the optimal effort in a repeated moral hazard problem without persistence in which the marginal disutility of effort is $(1-\beta(1-\delta))$. This related moral hazard problem can be thought of as an auxiliary problem to ours, since it can

\footnotetext{
${ }^{2}$ Without loss of generality, it is assumed that $s_{0}=0$.
} 
be easily solved and used to learn about the properties of the solution to the problem with persistence.

Denote the solution for effort in the auxiliary problem as $\left\{\tilde{e}_{t}\right\}_{t=1}^{\infty}$. The sequence of optimal contingent recommendations for $s$ in the problem with persistence will be the same as the recommendations for $\tilde{e}$ in the related repeated moral hazard, and the sequence of contingent consumption will be exactly the same in both problems. Using the definition of $s$ we can recover the corresponding sequence of effort levels for the problem with persistence:

$$
\begin{aligned}
e_{1}= & s_{1}=\tilde{e}_{1}, \\
e_{2}= & s_{2}-(1-\delta) s_{1}=\tilde{e}_{2}-(1-\delta) \tilde{e}_{1} \\
& \vdots \\
e_{t}= & s_{t}-(1-\delta) s_{t-1}=\tilde{e}_{t}-(1-\delta) \tilde{e}_{t-1} \quad \ldots
\end{aligned}
$$

In a repeated moral hazard the sequence of required effort will typically be fairly smooth. This, together with the equivalence just established, implies that, under the assumption of linear disutility of effort, if there is persistence it is optimal for the principal to ask the agent to make most of the effort in the first period. If $\delta$ is small enough, the agent will be asked to accumulate a given certain level of human capital, and in the following periods he will simply have to provide little increments to compensate for the tapering off of the effect of the first effort.

Note that, as in any repeated moral hazard problem with linear disutility of effort, absent a lower bound on effort, the auxiliary problem may have either a trivial solution, in which 
the agent always deviates and chooses a very negative level of $\tilde{e}$, or no solution, if we were considering a social planner's problem in which negative effort is the most efficient way of providing the agent with utility. The standard approach in the literature is to impose a non negativity constraint on per period effort: $\tilde{e}_{t} \geq 0$ for all $t .^{3}$ To preserve our equivalence result, the corresponding restriction in our original problem with persistence is $s_{t} \geq 0$ for all $t$. This guarrantees that the conclusions from our analysis do not apply only to a vacuous class of problems. ${ }^{4}$

From the set of equations in (1) we see that drops in the value of $s$ from one period to the next could imply negative effort levels; the non negativity constraint on $s$, however, imposes an endogenous lower bound on $e$. Also, we could always find examples for which effort stays in positive values. Any problem for which the sequence of $\tilde{e}_{t}$ is bounded above and below can be mapped into a persistence problem for which depreciation is high enough as to never imply negative effort.

An important implication of the relation we established between the problem with persistence and the auxiliary one is that the sequence of contingent consumption will be exactly the same in both problems. This makes the two problems observationally equivalent. The results found in the moral hazard literature about the long run distribution of utilities and the individual consumption paths will also hold in our environment with persistence and linear

\footnotetext{
${ }^{3}$ For some examples of papers that assume linear disutility of effort, see [1], [4], [7], or [9].

${ }^{4}$ We thank an anonimous referee for pointing out this problem in an earlier version of the paper.
} 
disutility. $^{5}$

The more general case of convex disutility of effort would differ from the one studied here mainly because the marginal disutility of effort that the agent equates throughout the periods varies when considering a deviation. The closer the disutility is to linear, however, the less important the change in marginal disutility, and the more the contract will resemble the one described here.

\section{Conclusion}

The moral hazard literature has pointed out repeatedly the importance of generalizing the current models of asymmetric information to setups in which either the hidden endowment of the agent or the effect of the agent's effort are correlated in time. In this note we find a particular modelling of temporal persistence of the agent's effort that allows us to very simply characterize the optimal contract. Under the assumption of linear disutility of effort, we show that when output depends on a distributed lag of past efforts the optimal contingent consumption is identical to the one obtained in a related moral hazard problem without persistence. We conclude that persistence does not necessarily change the properties of the optimal contract.

\footnotetext{
${ }^{5}$ See $[10],[8],[6]$, and [2].
} 


\section{References}

[1] Atkeson, A. "International Lending with Moral Hazard and Risk of Repudiation", Econometrica, 59 (1991), 1069-1089.

[2] Atkeson, A. and R. E. Lucas, Jr, "Efficiency and Equality in a Simple Model of Efficient Unemployment Insurance". J. Econ. Theory 66 (1995), 64-88.

[3] Fernandes, A. and C. Phelan, A Recursive Formulation for Repeated Agency with History Dependence. J. Econ. Theory 91 (2000): 223-247.

[4] Hopenhayn, H. and J.P. Nicolini: "Optimal Unemployment Insurance", Journal of Political Economy, 105 (1997), 412-438.

[5] Mukoyama, T. and A. Sahin, Repeated Moral Hazard with Persistence, Forthcoming in Economic Theory (2004)

[6] Phelan, C., Repeated Moral Hazard and One-Sided Commitment. J. Econ. Theory 66 (1995), 468-506.

[7] Phelan, C., "Incentives, insurance, and the variability of consumption and leisure" Journal of Economic Dynamics and Control 18, Issues 3-4, (1994), Pages 581-599.

[8] Phelan, C. and R. M. Townsend, Computing Multi-Period, Information-Constrained Optima. Rev. Econ. Studies (1991) 58, 853-881. 
[9] Shavell, S. and L. Weiss: "The Optimal Payment of Unemployment Insurance Benefits over Time", Journal of Political Economy, 87 (1979), 1347-1362.

[10] Spear,S. E. and S. Srivastava, On Repeated Moral Hazard with Discounting. Rev. Econ. Studies 54 (1987), 599-617. 